\section{P0-0544 VOLUNTARY TOXOPLASMOSIS SCREENING IN PREGNANCY UNDERESTIMATES ACTIVE INFECTION IN DEPENDENCY OF SOCIOECONOMIC FACTORS: DATA FROM A POPULATION-BASED SURVEY OF NEONATES IN POMERANIA (SNIP)}

${ }^{1}$ A Lange, ${ }^{2} \mathrm{JR}$ Thyrian, ${ }^{2} \mathrm{~W}$ Hoffmann, ${ }^{3} \mathrm{M}$ Zygmunt, ${ }^{4} \mathrm{HN}$ Lode, ${ }^{5} \mathrm{M}$ Heckmann ${ }^{1}$ Neonatology and Pediatric Intensive Care, University of Greifswald, Greifswald, Germany; ${ }^{2}$ Institute of Community Medicine Division of Health Care Epidemiology and Community, University of Greifswald, Greifswald, Germany; ${ }^{3}$ Department of Gyaecology and Obstetrics, University of Greifswald, Greifswald, Germany; ${ }^{4}$ Department of Pediatrics, University of Greifswald, Greifswald, Germany; ${ }^{5}$ Department of Pediatrics Neonatology and Pediatric Intensive Care Medicine, University of Greifswald, Greifswald, Germany

10.1136/archdischild-2014-307384.1187

Background and purpose $40 \%$ of all initial infections with Toxoplasma gondii during pregnancy develop into connatal toxoplasmosis, which is associated with the risk of severe damage to the CNS, frequent premature deliveries, and stillbirths. In Germany, toxoplasmosis immunity screenings are not covered by the state health insurance as a general preventive care service, in contrast to screening for rubella immunity. Therefore, we analysed the impact of socioeconomic factors on the efficiency of private toxoplasmosis screening during pregnancy in a population-based study.

Material and methods Toxoplasmosis and rubella screening data were collected from 5403 mothers during the period from May 2002 to June 2008 within the population-based Survey of Neonates in Pomerania (SNiP).

Results At the first screening, 34.2\% of expecting mothers were immune to toxoplasmosis, $75.2 \%$ had immunity against rubella after active immunisation. Negative immunity for toxoplasmosis was found in $39.7 \%$ and in $7.7 \%$ for rubella (missing information: toxoplasmosis: $25.8 \%$; rubella $17.2 \%$ ). Less than $10 \%$ of the women without immunity participated in a second toxoplasmosis screening. An active toxoplasmosis infection was found in $0.3 \%(\mathrm{n}=17)$ women during pregnancy. There were no infections with the rubella virus. Pregnant women with a higher socioeconomic status participated in screenings considerably more frequently.

Conclusion Our population-based sample demonstrated a substantial risk of toxoplasmosis infection during pregnancy in contrast to the rubella risk. Furthermore, lower socioeconomic status adversely affects the rate of toxoplasmosis screening. This data support the need for toxoplasmosis screening for pregnant women as a general health care benefit covered by insurance.

\section{PO-0545 INFECTIOUS DISEASES IN TERM NEWBORN INFANTS}

E Lekic, E Lekic, R Rudanovic, U Dragas. Center of Neonatology, Institute for Children's Diseases, Podgorica, Montenegro

\subsection{6/archdischild-2014-307384.1188}

Backgraund and aims Maternal, environmental, and host factors determine which infant exposed to a potentially pathogenic organism will develop serious or other potentially invasive infections, causing significant mortality and long-term morbidity in neonates. The purpose in this retrospective study was to identifications the bacterial microorganisms caused neonatal infectious diseases in term newborns hospitalised in the Centre of Neonatology, during the period of 2002, 2003 and 2004.

Methods We used clinical, microbiological, laboratory and radiology methods.
Results 1391 term newborns (TNB) were treated at the Centre of Neonatology in Podgoric during the period 2002-2004. In 528 TNB were proven infections. Most frequent infectious diseases were :omphalitis (44,9\%), pneumonia (18,5\%), sepsis $(10,9 \%)$, cutaneus infections $(8,7 \%)$, urinary tract infection $(5,3 \%)$, conjunctivitis $(5,5 \%)$, otitis media $(3,8 \%)$, mastitis $(1,7 \%)$, diarrhoea $(0,7 \%)$. Sepsis and/or meningitis were diagnosed in 58 term newborn $(10,9 \%)$. The bacterial agents responsabile for sepsis and/or meningitis were: Coagulasa -Negative Staphylococcus (41,3\%), Staphylococcus Aureus (19\%), E.coli (5.3\%), then with equally frequency SGB, SGA, Streptococcus pneumoniae, Enterococcus, L. Monocytogenes, Klebsiella pneumoniae, Acinetobacter, Serratia, Pseudomonas, Klebsiella-Enterobacter (each one 1,7\%). Meningitis were proven in $16 \mathrm{TNB}$ or $27,6 \%$.

Conclusions Temporal and geograpgic differences of various neonatal patogens are well recognised. It is important to identifications the bacterial microorganismsims in our region, analysis of longitudinal trends assist in the formulations of strategies to treat and prevent neonatal serious infections.

\section{PO-0546 LESSONS FROM 10 YEARS OF INVASIVE FUNGAL INFECTION AT SINGAPORE GENERAL HOSPITAL NICU: EPIDEMIOLOGY, RISKS, CLINICAL COURSE AND OUTCOMES}

${ }^{1} \mathrm{DJ}$ Leo, ${ }^{2} \mathrm{PS}$ Liew, ${ }^{2} \mathrm{~S}$ Arunachalam, ${ }^{2} \mathrm{WB}$ Poon. ${ }^{1}$ Paediatric Medicine, KK Women's and Children's Hospital, Singapore, Singapore; ${ }^{2}$ Neonatal and Developmental Medicine, Singapore General Hospital, Singapore, Singapore

\subsection{6/archdischild-2014-307384.1189}

Introduction Invasive fungal infections (IFI) in preterm neonates are rare, but associated with increased mortality and morbities, both short and long term. IFIs in preterms are also increasing globally.

Aims To review the epidemiology, risk factors, clinical course and outcomes of all IFIs in newborns at Singapore General Hospital for the period $1 / 1 / 2002$ to $31 / 7 / 2012$.

Methods Retrospective matched case-control study. Cases of IFI were matched 1:1 with another neonate of the same gestation in the same year of birth.

Results 6 cases of IFI were matched with 6 controls. All were extremely low birth weight (ELBW) infants. Many IFI cases had risk factors for sepsis perinatally.

Significant risk factors associated with IFI include surgery (OR 25.000 95\% CI 1.200-520.734), more than 35 days of conventional ventilation (OR 25.000 95\% CI 1.200-520.734), 9 lots of platelet transfusion or more (OR 7.000 95\% CI 1.140 42.969), vancomycin (OR 25.000 95\% CI 1.200-520.734) and cephalosporins (OR 6.000 95\% CI 1.003-35.908).

5 out of 6 IFI presented with thrombocytopenia with elevated C-reactive protein. Other presentations included hypotension (3 out of 6 ), increased ventilation settings (2 out of 6 ) and leucopenia (2 out of 6 ).

All IFIs were Candida albicans. Multiorgan involvement is prevalent. All 6 IFIs had positive endotracheal as well as urine cultures. 3 had positive catheter tip cultures, 2 had positive peritoneal fluid cultures, and 1 had positive blood culture.

Conclusion Classical presentations and significant risk factors for IFI in ELBWs were identified which allowed earlier identification and possible modification of risks. 